\title{
Verifiable Observation of Permissioned Ledgers
}

\author{
Ermyas Abebe*, Yining Hu*, Allison Irvin*, Dileban Karunamoorthy*, Vinayaka Pandit*, \\ Venkatraman Ramakrishna*, Jiangshan $\mathrm{Yu}^{\dagger \uparrow}$ \\ *IBM Research \\ ${ }^{\dagger}$ Monash University
}

\begin{abstract}
Permissioned ledger technologies have gained significant traction over the last few years. For practical reasons, their applications have focused on transforming narrowly scoped use-cases in isolation. This has led to a proliferation of niche, isolated networks that are quickly becoming data and value silos. To increase value across the broader ecosystem, these networks must seamlessly integrate with existing systems and interoperate with one another. A fundamental requirement for enabling crosschain communication is the ability to prove the validity of the internal state of a ledger to an external party. However, due to the closed nature of permissioned ledgers, their internal state is opaque to an external observer. This makes consuming and verifying states from these networks a non-trivial problem.

This paper addresses this fundamental requirement for state sharing across permissioned ledgers. In particular, we address two key problems for external clients: (i) assurances on the validity of state in a permissioned ledger and (ii) the ability to reason about the currency of state. We assume an adversarial model where the members of the committee managing the permissioned ledger can be malicious in the absence of detectability and accountability. We present a formalization of the problem for state sharing and examine its security properties under different adversarial conditions. We propose the design of a protocol that uses a secure public ledger for providing guarantees on safety and the ability to reason about time, with at least one honest member in the committee. We then provide a formal security analysis of our design and a proof of concept implementation based on Hyperledger Fabric demonstrating the effectiveness of the proposed protocol.
\end{abstract}

Index Terms-Distributed Systems, Distributed Ledgers, Permissioned Networks, Blockchain, Interoperability, Applied Cryptography

\section{INTRODUCTION}

Permissioned networks are designed to address the needs of enterprises collaborating across organizational boundaries using a shared ledger [33], [23], [31], [51], [49]. They restrict membership to a known group of identities to keep data private and confidential. Unlike permissionless networks, the security in these systems relies on the ability to detect and hold parties accountable for their actions. The use of a shared ledger between enterprises can reduce counter-party risk and mitigate the need for costly and time-consuming dispute resolution processes, which often involves legal and judicial systems.

For practical reasons, the adoption of permissioned ledgers has thus far been driven through use-cases. Enterprises have been coalescing into consortia to create networks that address narrow use-cases in isolation. These use-cases often represent

ฯ Corresponding author. Email: jiangshan.yu@monash.edu one part of a complex business process. For example, various networks have emerged that focus on provenance [55], [26], [52], trade logistics [54] and trade-finance [56], [42], [45], all of which address different aspects of the broader process of global trade. This has led to a proliferation of niche and isolated networks that are quickly becoming data and value silos. To increase value across the broader ecosystem, permissioned networks must be able to securely communicate with other networks while integrating with existing legacy applications.

However, the restrictive nature of a permissioned network introduces two key challenges to an external observer: (i) the inability to verify that state shared externally is the same as the state agreed on internally and (ii) the inability to reason about the currency of the state as a result of having no visibility on the chain's progress. An ostensible solution to these problems is for the external client to query one or more parties in the network and obtain a set of notarized responses [7], [20]. However, in the absence of a system for detectability and accountability outside of the network, a malicious committee can choose to misrepresent facts by either presenting false or stale state.

This paper addresses the problem of state sharing across permissioned networks. We assume an adversarial model where all members of a committee can be malicious except for a single honest party. We rely on the fact that a single honest member can hold parties accountable if it is able to detect any misrepresentation of facts with external clients. In our approach, the permissioned network publishes commitments representing snapshots of its internal state at fixed intervals to a trusted third-party such as a secure public ledger. These commitments enable both honest members to verify state snapshots while raising disputes when conflicts are detected, and external clients to verify proofs against valid commitments according to a common notion of time. The process of resolving disputes-which in practice can take various forms such as fines, termination of membership in the network, or legal proceedings - is out of scope of this paper.

Related Work. The problem of a client needing to verify the state of a blockchain without directly observing it is not new. Several light client implementations [40], [41], [50] provide efficient ways to verify the inclusion of transactions in a ledger assuming partial access to the ledger data, such as block headers. More recently, several non-custodial Layer- 
2 protocols [29] that rely on the parent chain for security have become popular. Examples of these systems include Plasma [4], NOCUST [38], Optimistic Rollups schemes such Arbitrum [36] and ZK-Rollups schemes such as zkSync [47], [6]. Although these systems allow transactions to take place off-chain, their state is periodically check-pointed on the parent chain for settlement. These approaches rely on external users and watch services having access to the off-chain state in order to keep the operators honest, using a challengeresponse game. Cross-chain communication protocols such as Cosmos [43], Polkadot [57], Cardano [2], Ren [5] and BTC Relay [1] rely on a network of intermediate nodes having partial access to a chain's data in order to ensure validity of the state communicated. In contrast to these systems, our work assumes that an external client has no visibility of the internals of a permissioned ledger, including data such as block headers. A simplified approach [7], [20] to this problem requires an external client placing significant trust on the committee in order to rely on their claims. Alternatively, schemes such as ZK-SNARKS [15], ZK-STARKS [14] and Bullet-Proofs [18] are too complex and expensive approaches for addressing a range of general purpose uses cases that permissioned ledgers are designed for. Furthermore, it would be impractical to migrate today's real-world deployments to these schemes. See Appendix A for further discussion.

Contributions. To summarize, this paper makes the following contributions: (i) A formalization of the problem of state sharing across permissioned ledgers, first introducing a formal model of permissioned ledgers and then presenting a formal model for state sharing, along with their security properties in different adversarial conditions. To the best of our knowledge, this is the first attempt at providing a formal treatment of this problem. (ii) The design of a protocol for state sharing based on the formal model. The protocol uses a secure public ledger that acts as a bulletin board for state commitments and a global clock for synchrony. We examine the protocol's safety and liveness properties in the presence of at least one honest member in the network. (iii) A security analysis of the protocol proving the stated properties. (iv) A proof-ofconcept implementation and evaluation of the protocol using Hyperledger Fabric [33].

The remainder of the paper is organized as follows. Section II formalizes state sharing in permissioned networks and examines their security properties. Section III proposes and discusses the design of a protocol for state sharing that is secure against a malicious but cautious committee. Section IV discusses a proof of concept implementation based on Hyperledger Fabric with security analysis. Section V provides an evaluation of the protocol and, finally, Section VI offers concluding remarks.

\section{Formalizing State Sharing in PeRmissioned NETWORKS}

In this section we present a formal treatment of state sharing from a permissioned ledger to external parties. We first identify the parties involved and the different adversary models to consider. We then formalize essential properties of permissioned ledgers, and use these to describe the problem of state sharing. We do this by distinguishing between the internal state of a ledger, which is visible to parties within a network, and external views of a ledger, which are the observations that external parties can make. We introduce algorithms for generating and verifying proofs about the validity of internal state and show how these can be adapted to prove validity of state against one or more external views, thus enabling external clients to verify state independently. Using these constructs, we present formally the security guarantees that state sharing mechanisms must provide under different adversary models.

\section{A. System model}

Environment. In the communication between a permissioned ledger and external entities, we consider three main types of participants, namely internal clients, members of the management committee, and external clients. The management committee has full knowledge of the ledger and is responsible for its maintenance. Internal clients do not have direct visibility or direct access to the ledger but interact with the management committee to query and update states according to defined policies. Both the management committee and internal clients are considered participants of the permissioned network. External clients, on the other hand, are not participants of the network, but still have a need to obtain and verify states from the permissioned network.

Adversary model. We consider three types of adversary scenarios with respect to the management committee of a permissioned blockchain, as follows.

- Trustworthy committee. Some members of the management committee can be faulty. However, the committee as a whole is trustworthy as it can tolerate faults when making agreements.

- Malicious but cautious committee. The management committee may be malicious but cautious. It will behave arbitrarily when its malicious behaviors cannot be detected and are not accountable.

- Malicious committee. The management committee is malicious. It will behave arbitrarily with no other considerations.

Internal clients in a permissioned network generally assume a trustworthy committee. While they trust the management committee as a whole, they may not trust any individual member. External clients, on the other hand, have different levels of trust in the management committee of a permissioned ledger depending on the adversary model. While in some cases an external client may trust the consensus agreement of a permissioned chain (i.e. trustworthy committee), in many cases such a strong trust relationship cannot be assumed. This is largely because external clients do not have full visibility into the ledger and might not have reliable affiliations with internal participants of the network. Conversely, as permissioned ledgers are often maintained by a reputable consortium, it is unlikely that the entire management committee of a 
permissioned ledger is fully malicious. Thus, we focus instead on the more practical adversary scenario: the scenario where the management committee is malicious but cautious.

We assume that the permissioned ledger has sufficiently strong internal consensus mechanisms to thwart malicious behaviors from a predefined threshold of members of the management committee. We also assume that at least one member of the management committee is honest in its interactions with external clients. If no such member exists, the management committee is considered fully malicious and the ledger is deemed completely unreliable.

\section{B. Modeling permissioned ledgers}

We define a permissioned ledger as a state machine where the transition of states is processed by executing a sequence of transactions. The state machine is replicated and maintained by the management committee. The committee processes transactions and updates system states according to defined policies.

Definition 1: A permissioned ledger with $n$ state transitions is a tuple $\mathcal{L}^{n}=\left(\mathcal{T}_{i}, \mathcal{A}_{i}, \mathcal{M}_{i}, \mathcal{P}_{i}\right)_{i=1}^{n}$ of sets $\mathcal{A}_{i} \in \mathcal{A}$ of application states, sequences $\mathcal{T}_{i} \in \mathcal{T}$ of transactions, management committees $\mathcal{M}_{i} \in \mathcal{M}$, and management policies $\mathcal{P}_{i} \in \mathcal{P}$. Here, $\mathcal{A}$ refers to the set of all possible application states, $\mathcal{T}$ refers to the set of all possible subsequences of the transactions recorded on the ledger, $\mathcal{M}$ refers to the set of all possible management committees, and $\mathcal{P}$ refers to the set of all possible management policies. We denote by $\mathcal{S}_{0}$ the genesis state and by $\mathcal{S}_{n}$ the current state of $\mathcal{L}^{n}$.

Let $\mathcal{S}_{i} \in \mathcal{S}$ be the $i$-th state of $\mathcal{L}^{n}$ for some $i \in[1, n]$. Here, $\mathcal{S}$ refers to the set of all possible ledger states. Each state $\mathcal{S}_{i}$ is a tuple $\left(\mathcal{A}_{i}, \mathcal{M}_{i}, \mathcal{P}_{i}\right)$ of a set $\mathcal{A}_{i}$ of application states, a management committee $\mathcal{M}_{i}$, and a management policy $\mathcal{P}_{i}$. A transaction applied to state $\mathcal{S}_{i}$ updates one or more application states, the management committee, or the management policy from its current state $\mathcal{S}_{i}$ to the resulting state $\mathcal{S}_{i+1}$. We use $A_{i, j} \in \mathcal{A}_{i}$ to denote the $j$-th application state at state $\mathcal{S}_{i}$. Similarly, $T_{i, j} \in \mathcal{T}_{i}$ denotes the $j$-th transaction at state $\mathcal{S}_{i}$. We denote by $\mathcal{L}_{i} \in \mathcal{L}$ the $i$-th tuple $\left(\mathcal{T}_{i}, \mathcal{S}_{i}\right)$ of $\mathcal{L}^{n}$.

The application states take the form of either (i) a set of UTXOs or (ii) a set of key/value pairs in the case of an account model. These states are application dependent and can represent arbitrary data or assets. We assume that all transactions in a ledger are totally ordered. In practice the sequence $\mathcal{T}_{i}$ of transactions can represent a single transaction in some protocols [23] or a block of transactions in others [33], [31]. The management committee $\mathcal{M}_{i}$ represents the set of members responsible for state $\mathcal{S}_{i}$ according to the policy $\mathcal{P}_{i}$.

We define a pair of algorithms (StateGen, StateVer). Given a state and a sequence of transactions to be applied on this state as input, StateGen outputs a new state. The correctness of this transition can be verified by StateVer.

Definition 2: State transition and verification of ledger $\mathcal{L}^{n}$ is accomplished by a pair of algorithms (StateGen, StateVer) such that for all $i \in[1, n]$, we have
- StateGen $: \mathcal{T} \times \mathcal{S} \rightarrow \mathcal{S}$

- StateVer : $\mathcal{T} \times \mathcal{S} \times \mathcal{S} \rightarrow$ True/False

and

$\forall i \in[1, n], \operatorname{StateVer}\left(\mathcal{T}_{i}, \mathcal{S}_{i-1}, \operatorname{StateGen}\left(\mathcal{T}_{i}, \mathcal{S}_{i-1}\right)\right)=$ True

If all state transitions are valid, then we say the ledger is a valid ledger.

Definition 3: $\mathcal{L}^{n}$ is a valid ledger iff

$$
\forall i \in[1, n], \operatorname{StateVer}\left(\mathcal{T}_{i}, \mathcal{S}_{i-1}, \mathcal{S}_{i}\right)=\text { True }
$$

\section{Modelling state sharing}

To formalize a framework for state sharing of permissioned ledgers, we first distinguish between internal state and an external view of a ledger $\mathcal{L}^{n}$. The internal state is shared by all internal parties whereas the external view is the observation that external parties can make about the ledger. The management committee is responsible for maintaining both the internal state and the external view, and while all internal parties see the same ledger, there can be multiple different external views. This could be because the permissioned network chooses to expose a partial view of its ledger to different parties in the external world.

To prove the validity of a fact $F$ on a ledger $\mathcal{L}^{n}$, we introduce the notion of an assertion. An assertion is a verifiable proof against the internal state of the ledger that can be used to validate $F$. To offer external parties a similarly verifiable proof of facts against their view of the ledger, we need to adapt the construct of an assertion to external views. We do this through the notion of a wrapping function on an assertion. We use these three key constructs of views, assertions and wrappings to formalize state sharing in permissioned ledgers. Specifically, a proof of state system for permissioned ledger $\mathcal{L}^{n}$ comprises three pairs of algorithms, namely assertion generation and verification (PGen, PVer), view generation and verification (VGen, VVer) and wrapping (WPGen, WPVer). We formally define and expound on each of these in this subsection.

For a given ledger, $\mathcal{L}^{n}$, we denote a digest $\mathcal{D}_{i} \in \mathcal{D}$ as a unique representation of the state $\mathcal{S}_{i}$ and the set of facts in the ledger as $\mathcal{F}_{i} \in \mathcal{F}$, where $\mathcal{F}_{i}=\mathcal{T}_{i} \cup \mathcal{A}_{i} \cup \mathcal{M}_{i} \cup \mathcal{P}_{i}$. A verifiable assertion about any fact $F$ is generated by the management committee and is denoted as $\pi \in \Pi$. An assertion offers a verifiable proof that a fact is included in a ledger according to the policies of the management committee. For instance, if the system states of $\mathcal{L}^{n}$ are validated by the management committee by reaching an agreement via the PBFT [22] protocol, then the assertion $\pi$ can be a proof of membership that an application state $A_{i, j} \in \mathcal{A}_{i}$ is recorded on the ledger, signed by at least $2 f+1$ management committee members, where $f$ is the number of faulty nodes the system can tolerate.

Definition 4: Assertion generation and verification of ledger $\mathcal{L}^{n}$ on a fact is a pair of algorithms (PGen, PVer) such that

- PGen : $\mathcal{F} \times \mathcal{D} \times \mathcal{M} \times \mathcal{P} \rightarrow \Pi$

- PVer : $\mathcal{F} \times \Pi \times \mathcal{D} \rightarrow$ True/False 
and we have that

$\forall i \in[1, n], F \in \mathcal{F}, \operatorname{PVer}\left(F, \operatorname{PGen}\left(F, \mathcal{D}_{i}, \mathcal{M}_{i}, \mathcal{P}_{i}\right), \mathcal{D}_{i}\right)=$ True

Since an assertion that is valid at state $S_{i}$ may not be valid at state $S_{i+1}$, internal clients should have the ability to use an assertion $\pi$ to not only verify that a fact is a part of the ledger, but also that it is correct at a given point in time. However, because clients do not have a direct view of states of a permissioned ledger, we need a mechanism to bridge the view between clients and the management committee. This is done through a deterministic function, $\tau(i)$ that maps a state $\mathcal{S}_{i}$ of the ledger $\mathcal{L}^{n}$ to a unique time point on a globally synchronized clock, where $i \in[1, n]$.

Definition 5: For a ledger $\mathcal{L}^{n}$, an assertion $\pi$ on a fact $F$ is valid at state $\mathcal{S}_{i}$ iff

$$
\exists i \in[1, n], \operatorname{PVer}\left(F, \pi, \mathcal{D}_{i}\right)=\text { True }
$$

and we say $F$ is current at time $t$ iff $F$ is valid at $\mathcal{S}_{j}$ such that

$$
\begin{cases}\tau(j) \leq t<\tau(j+1), & j \in[1, n-1], \\ \tau(j) \leq t & j=n .\end{cases}
$$

To provide a verifiable proof to external clients, the management committee must release a commitment about the internal state of the ledger to the external world. This commitment, denoted as $V \in \mathcal{V}$, forms the external view and is defined through a pair of algorithms (VGen, VVer).

Definition 6: View generation and verification of ledger $\mathcal{L}^{n}$ on a state is a pair of algorithms (VGen, VVer) such that

- VGen : $\mathcal{D} \times \mathcal{M} \times \mathcal{P} \rightarrow \mathcal{V}$

- VVer : $\mathcal{D} \times \mathcal{M} \times \mathcal{P} \times \mathcal{V} \rightarrow$ True/False

and we have that

$$
\begin{aligned}
\forall i \in[1, n], V \in \mathcal{V}, \operatorname{VVer} & \left.\mathcal{D}_{i}, \mathcal{M}_{i}, \mathcal{P}_{i}, \operatorname{VGen}\left(\mathcal{D}_{i}, \mathcal{M}_{i}, \mathcal{P}_{i}\right)\right) \\
& =\text { True. }
\end{aligned}
$$

Since we can have multiple valid external views of a permissioned ledger, we denote an external view $\mathcal{V}_{i}^{k} \in \mathcal{V}$ on $\mathcal{S}_{i}$ as the set of $k$ possible individual views $V_{i}^{1}, \ldots, V_{i}^{k}$ on $\mathcal{S}_{i}$. In practice, we expect that $\mathcal{V}_{i}^{k}$ is published by the management committee. It is possible that VGen is not deterministic and there exists multiple valid views on the same state. If all individual views of a state are the same, i.e. $k=1$, then we say there exists a consistent view of the ledger state.

Definition 7: The external view $\mathcal{V}_{i}^{k}$ on $\mathcal{S}_{i}$ of ledger $\mathcal{L}^{n}$ for some $i \in[1, n]$ is consistent if

$$
\forall x, y \in[1, k], V_{i}^{x}=V_{i}^{y} .
$$

If the external view is consistent for all $\mathcal{S}_{i}$, then we say the external view on $\mathcal{L}^{n}$ is consistent.

Since a view may or may not be the digest of a state, the system should be able to prove a fact against a released view. We define the proof of a fact against a view as a wrapping of the proof of the fact against the digest.

Definition 8: A wrapping for assertion generation PGen and verification PVer is a pair of algorithms (WPGen, WPVer) such that
- WPGen : $\mathcal{F} \times \Pi \times \mathcal{D} \times \mathcal{V} \rightarrow \mathcal{W}$

- WPVer : $\mathcal{F} \times \mathcal{W} \times \mathcal{V} \rightarrow$ True/False

and we have that $\forall i \in[1, n], F \in \mathcal{F}, \mathcal{D}_{i} \in \mathcal{D}, \mathcal{M}_{i} \in \mathcal{M}, \mathcal{P}_{i} \in$ $\mathcal{P}$ and

$$
\begin{gathered}
\operatorname{PGen}\left(F, \mathcal{D}_{i}, \mathcal{M}_{i}, \mathcal{P}_{i}\right)=\pi \wedge \operatorname{VGen}\left(\mathcal{D}_{i}, \mathcal{M}_{i}, \mathcal{P}_{i}\right)=V_{i} \Rightarrow \\
\operatorname{WPVer}\left(F, \operatorname{WPGen}\left(F, \pi, \mathcal{D}_{i}, V_{i}\right), V_{i}\right)=\text { True. }
\end{gathered}
$$

\section{Security properties}

As addressed previously, the management committee may be trustworthy, malicious but cautious, or fully malicious. We do not consider the case of a trustworthy management committee as the security guarantee of this case is fully dependent on the consensus protocol of the permissioned ledger. We say a system is Type- 1 secure if it is secure against a malicious attacker and Type-2 secure if it is secure against a malicious but cautious attacker.

Definition 9: A proof of state system for permissioned ledger $\mathcal{L}^{n}$ is Type-1 secure iff $\forall F \in \mathcal{F}, W \in \mathcal{W}, i \in$ $[1, n], V_{i} \in \mathcal{V}$, we have that

$$
\begin{aligned}
& \operatorname{WPVer}\left(F, W, V_{i}\right)=\text { True } \Rightarrow \\
& \exists \mathcal{D}_{i}, \mathcal{M}_{i}, \mathcal{P}_{i}, \text { s.t. } \operatorname{PGen}\left(F, \mathcal{D}_{i}, \mathcal{M}_{i}, \mathcal{P}_{i}\right)=\pi \wedge \\
& \operatorname{VGen}\left(\mathcal{D}_{i}, \mathcal{M}_{i}, \mathcal{P}_{i}\right)=V_{i} \wedge \\
& \operatorname{WPGen}\left(F, \pi, \mathcal{D}_{i}, V_{i}\right)=W .
\end{aligned}
$$

A malicious but cautious committee will misbehave if its actions cannot be detected. For a system to be resilient to such an adversary, honest parties must have knowledge of the system that enables them to detect and prove misbehavior. Different types of participants have different sets of knowledge. The internal client $(I)$ has knowledge of a set of facts $F$ and associated proofs $\pi$ through internal inquiry, and may or may not have knowledge of the digest $\mathcal{D}_{i}$ of a ledger state $\mathcal{S}_{i}$; the external client $(E)$ has knowledge of a set of $(F, W, V)$; and the members of the management committee $(M)$ have knowledge of $\mathcal{L}^{n}$. We use $K_{A}^{X}(d)$ to denote participants of type $A$ having knowledge of some data $d$, such that the participants are honest (if $X=H$ ) or rational (if $X=R$ ), where $A \in\{I, E, M\}$ and $X \in\{H, R\}$.

Definition 10: A proof of state system for permissioned ledger $\mathcal{L}^{n}$ is Type-2 secure if and only if the following two conditions hold $\forall \mathcal{F}^{*} \subseteq \mathcal{F}, \mathcal{W}^{*} \subseteq \mathcal{W}, \mathcal{V}^{*} \subseteq \mathcal{V}$ :

- $\forall F \in \mathcal{F}^{*}, W \in \mathcal{W}^{*}, V \in \mathcal{V}^{*}$, the system is Type-1 secure; and

- $\forall F \in \mathcal{F} \backslash \mathcal{F}^{*}, W \in \mathcal{W} \backslash \mathcal{W}^{*}, V_{i} \in \mathcal{V} \backslash \mathcal{V}^{*}$, $\operatorname{WPVer}\left(F, W, V_{i}\right)=$ True, we have that $\exists V_{i}^{\prime} \in V^{*}, A \in$ $\{I, E, M\}$ s.t. external view $V_{i}$ is not consistent with $V_{i}^{\prime}$ and $K_{A}^{H}\left(V_{i}, V_{i}^{\prime}\right)$.

\section{PROTOCOL}

This section provides an overview of the proposed protocol and parameters that influence its behavior. The protocol interfaces with three entities: (i) the management committee of a permissioned network (ii) an immutable public bulletin board, and (iii) external clients. The protocol consists of 
four sub-components: (i) generation and publication of views to the bulletin board, (ii) querying the bulletin board for a view corresponding to time $t$, (iii) querying a member of the management committee for a proof about facts in the ledger against the view, and (iv) the verification of proofs by the external client to establish the validity of facts. Two key parameters that influence the behavior of this protocol are (i) the number of members, $n$, of the management committee who choose to publish their state digest at any give instance and (ii) the interval, $k$, at which publications are made. These parameters represent trade-offs between safety, liveness, and cost as discussed in Section III-E. Figure 1 provides an overview of the protocol.

\section{A. Generation of State Digests}

For every new block at height $i$, each committee member computes a digest of the state $\mathcal{S}_{i}$ which comprises the set of application states $\left(\mathcal{A}_{i}\right)$, management committee members $\left(\mathcal{M}_{i}\right)$ and management policies $\left(\mathcal{P}_{i}\right)$. The digest is computed by updating an accumulator [16], a cryptographic primitive that can represent a set of elements in the form of a succinct digest and allows for proofs of membership and non-membership without revealing other information about the set. Various cryptographic accumulator schemes could be employed for this purpose, ideally allowing for efficient dynamic updates.

Given that all nodes have full visibility of the state in the ledger, and that the protocol offers deterministic finality [12], all members will compute the same digest for $\mathcal{S}_{i}$. Nodes then create a view $\mathcal{V}_{i}$ using function VGen (as defined in Def. 6) that consists of the digest $\mathcal{D}_{i}$, a signature over the digest and the corresponding block height $i$. A view is therefore defined as $\mathcal{V}_{i}=\left(\mathcal{D}_{i}, i, \operatorname{sig}_{\mathrm{m}}\right)$.

The protocol proceeds in rounds, wherein every $k$ blocks a new round is initiated. In each round $r$, a subset of members from the committee, $M_{i}^{r} \in \mathcal{M}_{i}$, can choose to publish their accumulator at the current block height $i$, to the bulletin board. The size of the subcommittee should at least be $f+1$ where $f$ is the failure threshold of the network, so as to ensure at least one member publishes in each round. A network can choose different strategies for publishing. Having all members publish in every round can be expensive, but allows external clients to quickly determine if a commitment has been seen by all members and is therefore safe. A deterministic selection of unique subsets of members on every round, such as a simple round robin assignment, trade-offs cost for delayed safety. See Section III-E for further discussion on the implications of selection strategies in relation to safety and liveness.

\section{B. Publishing Commitments}

Publishing views to an immutable bulletin board provides visibility to external clients and mitigates the potential for a network to equivocate on facts about the state of the ledger with external clients. The bulletin board uses a smart contract published by the permissioned network that is known to external clients a priori. The smart contract is bootstrapped with a policy specifying a list of public keys of the management committee. We assume establishing the identity and validity of public keys occurs off-chain, coordinated between external clients and management committees. The bulletin board performs functions that include tracking published views for accountability, ensuring that views do not conflict, and offering a mechanism by which parties can report conflicts.

Appendix B presents an algorithm describing how the bulletin board works. A publication to the bulletin board is a tuple $\left(V_{i}^{n}, H_{i}^{n}\right)$ where $V_{i}^{n}$ is the view at $L_{i}$ computed by subcommittee member $n$, and $H_{i}^{n}$ a rolling hash of all past views observed by $n$, up to $\mathcal{V}_{i}$. The rolling hash of views is trivially computed by each node on every new view generation by concatenating the hash of the previous accumulator with the previous rolling hash, and hashing the result. The rolling hash offers two benefits: (i) it serves as a mechanism by which parties implicitly validate views from previous rounds in which they did not participate (ii) it adds further accountability for the publishing party. If in a previous round another member published a false accumulator, and another member in a subsequent round provided a rolling hash which included that false accumulator, it is evident that the parties are colluding and can be held accountable.

\section{Detection and Reporting}

There are two scenarios in which an invalid commitment could be detected by a single honest management committee member $m_{h}$ :

i $m_{h} \in M_{i}^{r}$ : The honest member is part of the subcommittee publishing in the round. In this case, the member's accumulator will conflict with accumulator values submitted by malicious committee members or in the rolling hash of past accumulators. This inconsistency is detected by the bulletin board.

ii $m_{h} \notin M_{i}^{r}$ : The honest member is not publishing in the current round but observes events of publications by other members. If the member detects that a posted view does not match its accumulator for the ledger height, it reports the conflict to the bulletin board.

The delay in detecting and reporting conflicting commitments posted by a malicious member is the time before an honest member can detect and report view discrepancies. In the second model where members observe events from the bulletin board, this time is only influenced by network delays and crash failures.

The detection of a conflict and its reporting on the bulletin board enables the rest of the management committee and external clients to become aware of the presence of malicious behavior. However, such conflicts don't provide any information about the validity of a view, and by extension, which parties were malicious and which ones were honest. The process of resolving this dispute is to be handled out-ofband by the respective committee members. In practice, this could involve an external arbitrator. 


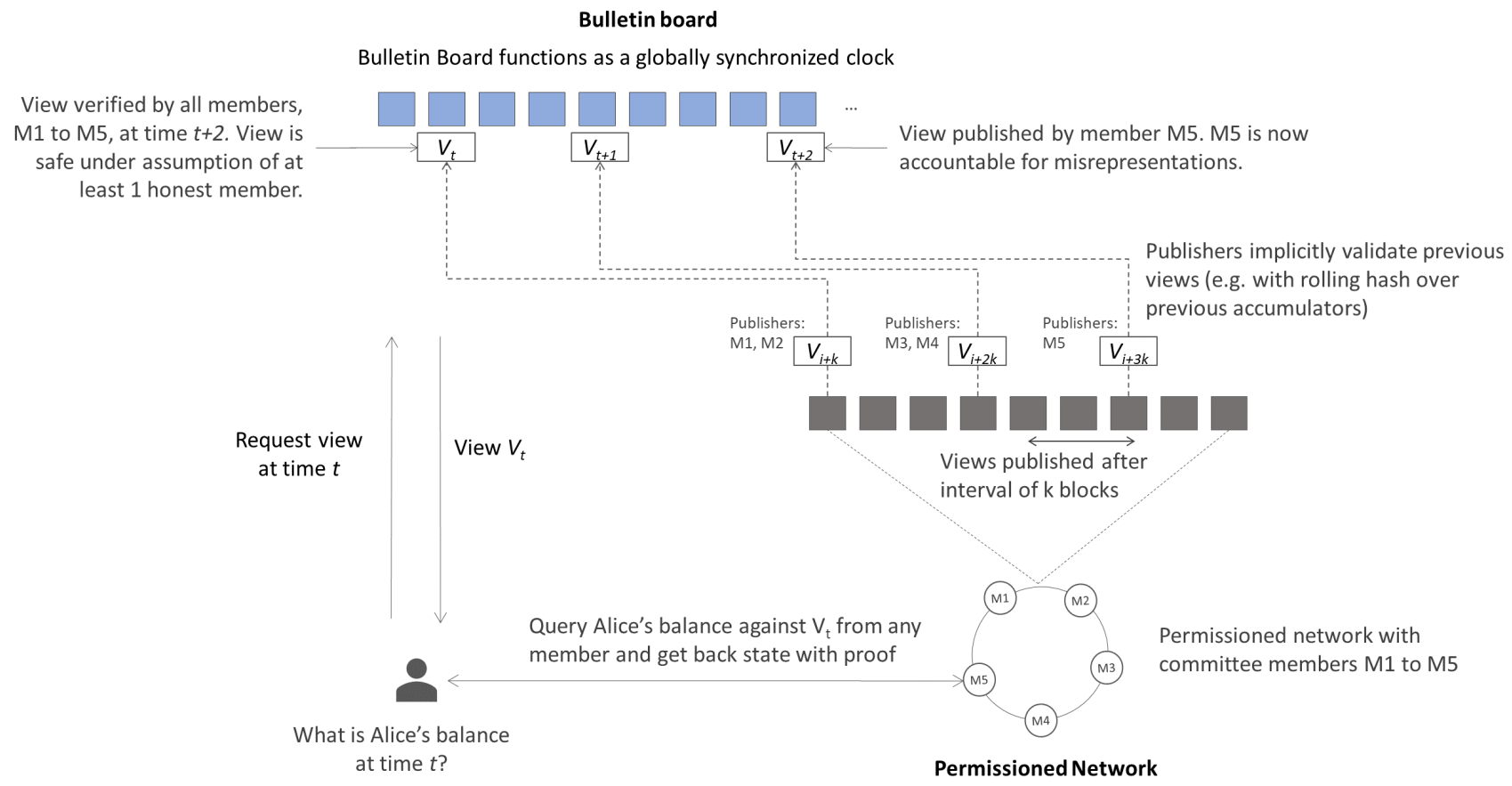

Fig. 1: Protocol overview: verifiable observations of state on permissioned ledgers.

\section{State Query and Verification}

External clients use the bulletin board as a source of views of the permissioned ledger. An external client will query the smart contract for views either seen by all members or views that were recently published. The bulletin board's block height allows the client to reason about the time at which publications were made. The client will use the view from the bulletin board to query a committee member for a given state (e.g. an application state $A_{i, j} \in \mathcal{S}_{i}$ ). The node will use the PGen algorithm to create an assertion $\pi$ that the application state $A_{i, j}$ is in the state set $\mathcal{S}_{i} . \pi$ includes the membership proof of the state in the accumulator.

\section{E. Discussion}

The proposed protocol offers guarantees of detectability of malicious behavior associated with state sharing and accountability of members responsible for the misbehavior, assuming at least one honest member in the network. In a permissioned context, this event would have legal and reputational ramifications that would serve as a deterrent for misbehavior by a malicious but cautious adversary.

However, a safety guarantee for the external client on a state commitment is only achieved when all members of the committee have implicitly or explicitly published the same accumulator for a given block height (see III-B). That is, it is explicit when it is a member of the subcommittee publishing in a given round, or implicit when, in a future round, it endorses a history of past accumulators of which that state commitment is a part. This however, affects liveness if the honest member is unavailable. More practically, an external agent can utilize a commitment without requiring a guarantee that all members have agreed on the same accumulator value, by relying on the fact that parties will be held accountable for misrepresenting state. For instance, an external agent that does not expect any collusion between parties can utilise a commitment as soon as it is published. Similarly, an agent that does not expect more than one third of nodes to collude can wait until more than a third of the members have published the same accumulator for a given block height. More generally, an external agent that assumes a colluding set of $c$ members in the network would need to wait for $c+1$ votes on a commitment.

The guarantee on currency of state is influenced by the intervals at which state commitments are published, expressed as the number of blocks $k$. Reducing $k$ increases the frequency of commitments and thus offers external clients better state coherence with the permissioned ledger. While this is desirable, it increases the overhead and cost of publishing commitments. In addition, this could also be constrained by the throughput of the bulletin board.

\section{Proof of Concept with Hyperledger FABRIC}

Hyperledger Fabric is an open-source permissioned blockchain designed for enterprises [10]. Fabric's design uses an execute-order-validate model for appending transactions to the blockchain. A Fabric network consists of an ordering service and one or more channels. Each channel consists of a set of peers, managed by independent organizations, and represents a unique ledger with its own genesis block. The ledger is based on the account model. A subset of peers run smart contracts and endorse transaction proposals submitted by clients. All peers validate blocks from the ordering service. 
We refer the reader to Fabric's protocol documentation [33] for details.

\section{A. Enabling state sharing in Hyperledger Fabric.}

We describe the implementation of the protocol presented in Section III for sharing state from a Fabric network to an external client. Fabric agents listen to block events [3] from peers and maintain a digest of the entire ledger state in the form of an RSA accumulator [16]. They publish the digest as a view to a contract on the Ethereum blockchain. External clients fetch views from the Ethereum contract and make a request for a state corresponding to the view from the Fabric agent. The agent retrieves the state from the Fabric ledger and generates a proof of membership of that state in the accumulator. The external client verifies the state using this proof.

Cryptographic accumulators for view generation. Our design uses a dynamic RSA accumulator [16], [21] to represent a succinct digest of the Fabric ledger. We make use of an opensource library from Westar Labs [35]. The RSA accumulator is set up with an RSA modulus $N$ that is the product of two safe primes $p$ and $q$. A base generator $g$ is selected from the set of quadratic residues modulo $N$. In order for each Fabric agent to create the same RSA accumulator, they use identical seeds to deterministically generate $p, q$ and $g$. For details on the construction of the accumulator and membership proofs see Appendix C.

Peer agents as generators of views. Each Fabric agent maintains an RSA accumulator as a digest of the complete state of the ledger using the DGen function described in Algorithm 2 in Appendix C. To do this, the agent subscribes to all block events from the peer, iterating through valid transactions and creating a list of the key/value pairs that are updated in the ledger, denoted the $K V$ write set [34]. Each $K V$ write is converted to a prime through the PrimeGen function as described in Appendix C. If the $K V$ write is marked with an "isDelete" flag, the element is deleted from the accumulator, otherwise it is added.

For each block height $i$, the Fabric peer agent also maintains a rolling hash, $H_{i}$, of the history of accumulators. The rolling hash is computed by hashing the concatenation of the previous rolling hash with the previous accumulator. The rolling hash enables the efficient verification of consistency of the accumulator between peer agents. For all peer agents to have calculated the same rolling hash for a block height they must have the same accumulator value for every previous block. Both the accumulator and the rolling hash are stored in a local persistent store of the Fabric agent. The agent storing prior accumulators for every block height allows proofs to be generated based on historical state.

Bulletin board. For a block height $i$, the agent publishes the accumulator, the rolling hash, and its signature to the Ethereum contract. We use Ethereum as the bulletin board for its desirable properties, which include a tamper-resistant log for non-repudiability, public availability, and support for smart contracts. A ManagementCommittee contract maintains a list of the public keys of agents of Fabric peers that make up the management committee. These public keys are recorded against the corresponding Ethereum address of the agent. The LedgerState contract accepts views submitted by Fabric agents. The contract only accepts views if the transaction signature corresponds to a valid Ethereum account recorded in the ManagementCommittee contract.

Proof generation and verification of facts. An external client obtains a view from the LedgerState contract by specifying a block number of the Ethereum chain. It uses the view to request a Fabric agent for a fact about the state of the ledger corresponding to that view, e.g. a key/value pair of some application state $A_{i, j}$. The Fabric agent queries the peer for all historical states of the application and finds the latest version that is present in the accumulator sent by the external client. The agent then generates a membership proof of the prime representation of that state in the accumulator. The external client verifies this membership proof in constant time.

\section{B. Security analysis.}

Loosely speaking, assuming the bulletin board guarantees consensus safety, then all honest participants, including members of the management committee and external clients, have a consistent view (as in Def. 7) of the same internal state. A misbehavior can lead to conflicting valid view of the same block height. In addition, the correctness and soundness of the proof generation and verification is guaranteed by the underlying accumulator. When the management committee tries to add a false commitment to the bulletin board, such behavior will be detected by any honest party with the knowledge of the correct commitment at the same block height. So, any honest party with the knowledge of a conflict can raise an alert on the misbehavior with evidence. This prevents attacks from a malicious but cautious committee.

Theorem 1: Under the strong RSA assumption, with a secure signature scheme, and the security of the bulletin board, the permissioned ledger $\mathcal{L}^{n}$ of the proposed protocol is Type- 2 secure.

Proof of Theorem 1 can be found in Appendix D.

\section{Performance Evaluation}

We compared the performance of our protocol with a Fabric network to show whether the Fabric commitment agent can keep up with the peer network and publish current state to the bulletin board as the ledger grows. We used maximum throughput (transactions per second, or TPS) and latency as evaluation metrics. Studies have revealed how Fabric performs in different conditions [53] and provided guidelines to engineer a Fabric network for high performance [27].

\section{A. Experimental Setup}

Since we wish to compare the processing ability of our protocol relative to that of Fabric, any Fabric setup will suffice to produce an estimate as long as both the base network and our protocol are measured in identical environments. We conducted tests on a single VM running a Fabric 2 network 
in Docker containers, using Hyperledger Caliper [32] for workload generation and throughput measurement. The VM was allocated 32 vCPUs (Intel(R) Xeon(R) Gold 6140 CPU @ 2.30GHz) and 64GB of memory. The Caliper installation was bound to Fabric version 2.1.0, running on the same VM as the Fabric network. We used two different networks for our experiments: a single-peer network running the Smallbank benchmark [9] and a more production-like three-organization network running a trade contract [25]. The source code is available online ${ }^{1}$.

Details of the two sets of networks and benchmark applications are as follow:

1) Basic Smallbank: This is the simplest possible Fabric network, with a single peer (using Go LevelDB) and a certificate authority (CA) in a single organization, and a single ordering node running in solo mode. We used the smallbank benchmark application [9] offered by Caliper, which simulates common bank transactions. The create_account function was selected to drive transaction load and the query (account balance) function for query load.

2) Trade: This is a more realistic network, with three peers (using CouchDB) and CAs across three organizations (representing exporter, importer, and regulator) and a fivenode Raft ordering service [25]. This network runs a trade contract that supports trade requests, acceptances, and views [24]. The requestTrade function was selected to drive transaction load and the GetTradeStatus function for query load.

For each network and benchmark set, we first determined the maximum throughput achievable on our test machine without the state commitment protocol components, by:

- Launching the network with Docker Compose and installing the benchmark contract, and

- Increasing the transaction submission rate in the Caliper workload configuration until the TPS fell instead of increasing (indicating saturation).

For each network configuration, transaction arguments were chosen to guarantee that there were no conflicting transactions within a block. Conflicts can occur when a transaction records it has read state from the ledger and a prior transaction in the block has altered that state. To avoid conflicts, our configuration specified that keys were only created and never updated.

\section{B. State Commitment Capacity}

First, each Fabric network was launched without our protocol and its baseline performance (maximum TPS) measured. Each was then restarted with protocol components, namely one Fabric agent (instrumented to record TPS and latencies) for each peer and an Ethereum test network as a bulletin board. We ran tests using the maximum throughput workload configurations. Table I shows the baseline platform and protocol throughputs, which are measures of their respective processing

\footnotetext{
${ }^{1}$ https://github.com/dlt-interoperability
}

capacities. (For Trade, the BPS and TPS are the minimum of the three agents' reported values.)

TABLE I: Transaction Processing Capacity

\begin{tabular}{|c|c|c|}
\hline Scenario & Basic Smallbank & Trade \\
\hline Num Tx & 40,840 & 13,240 \\
\hline Max Network TPS & 370 & 138 \\
\hline Protocol BPS & 27.8 & 12.1 \\
\hline Protocol TPS & 278.4 & 121.5 \\
\hline
\end{tabular}

The maximum throughput achievable on our test machine using the basic network configuration and benchmark application was 370 TPS. The commitment agent was able to achieve a maximum throughput of 278 TPS in comparison. (Note: each block contains the maximum of 10 transactions as configured; hence the TPS is 10 times the blocks per second, or BPS). This includes the overhead of publishing to the bulletin board every 120 blocks for the Smallbank application, which corresponds to roughly every 4.3 seconds. The gas cost of publishing each commitment to the Ethereum bulletin board was 203,514.

\section{Query Capacity}

To understand how responsive an agent is to queries from external clients, we performed multiple state and proof queries after every batch of 120 blocks. The average query response time observed by an external client was around $231 \mathrm{~ms}$. This included the overall process of first querying the Ethereum bulletin board for a commitment, then querying the Fabric agent for a state that corresponds to the commitment and finally verifying the returned proof of membership. This time was independent of the number of states that had been accumulated.

\section{Analysis and Improvements}

Our measurements revealed that the agent was roughly $24.8 \%$ slower than the peak TPS for the simplest benchmark application, Smallbank, and $12.3 \%$ slower than the maximum TPS for the Trade application. While these results suggest that under a high transaction volume the currency of published data cannot be guaranteed, in real-world enterprise networks we are likely to encounter networks and contracts that are much more complex than these benchmarks. In such scenarios, transactions of interest would occur at lower frequencies and hence it is likely that our agents will deliver adequate performance in practice. In addition, we found that different agents deployed for each organization in the Trade network delivered similar TPS, confirming that size of the management committee and the network have no impact on agent throughput.

Several improvements can be made to the implementation and configuration of the protocol to significantly enhance its performance. From an implementation perspective, basic optimisations can be made to the agent to reduce $\mathrm{I} / \mathrm{O}$ and compute overheads. In addition, the configuration of the protocol can be relaxed depending on real world requirements. For instance, some use cases may require less frequent publication of accumulators. Similarly, networks could also choose to only compute and publish digests for a subset of keys that are 
shared with external clients, as opposed to the entire ledger. Such variations can reduce the cost and the TPS requirements on the agent. Further, to optimise query performance, frequently queried keys and their associated proofs can be cached.

The commitment scheme employed in the protocol could also be improved. For instance, by consolidating the update of multiple keys into a single operation [17], the cost of updating accumulators can be significantly reduced. In addition, other commitment schemes [8], [58] could also be investigated in future work.

\section{CONCLUSION}

This paper addressed an important problem challenging external consumers of state of permissioned ledgers: verifying the validity of state and reasoning about its currency, assuming at least one honest member in the network. This work lays the foundation for enabling cross-chain communication across permissioned networks, as well as integrating permissioned networks with legacy enterprise applications. We provided a formalization of the problem of state sharing under different adversarial conditions and proposed the design of a protocol for state sharing with safety and liveness trade-offs. We also presented a security analysis supporting our protocol design. Our experiments and analysis with Hyperledger Fabric showed the viability of the protocol with a number of avenues for improving performance.

\section{ACKNOWLEDGEMENT}

This work was partially supported by the Australian Research Council (ARC) under project DE210100019. The authors would like to thank Yacov Manevich for his review and feedback.

\section{REFERENCES}

[1] BTC Relay: A bridge between the Bitcoin blockchain and Ethereum smart contracts, http://btcrelay.org/

[2] Cardano: decentralized public blockchain and cryptocurrency project., https://cardano.org/

[3] Hyperledger Fabric: Peer channel-based event services, https://hyperledger-fabric.readthedocs.io/en/release-2.2/peer_event_ services.html

[4] Plasma, https://docs.ethhub.io/ethereum-roadmap/layer-2-scaling/ plasma/

[5] Ren project, https://renproject.io/

[6] zkSync: Secure, scalable crypto payments, https://zksync.io/

[7] Abebe, E., Behl, D., Govindarajan, C., Hu, Y., Karunamoorthy, D., Novotny, P., Pandit, V., Ramakrishna, V., Vecchiola, C.: Enabling Enterprise Blockchain Interoperability with Trusted Data Transfer. In: Proceedings of the 20th International Middleware Conference (Industrial Track). pp. 29-35 (2019)

[8] Agrawal, S., Raghuraman, S.: Kvac: Key-value commitments for blockchains and beyond. In: International Conference on the Theory and Application of Cryptology and Information Security. pp. 839-869. Springer (2020)

[9] Alomari, M., Cahill, M., Fekete, A., Rohm, U.: The cost of serializability on platforms that use snapshot isolation. In: 2008 IEEE 24th International Conference on Data Engineering. pp. 576-585 (2008)

[10] Androulaki, E., Barger, A., Bortnikov, V., Cachin, C., Christidis, K., De Caro, A., Enyeart, D., Ferris, C., Laventman, G., Manevich, Y., et al.: Hyperledger fabric: a distributed operating system for permissioned blockchains. In: Proceedings of the Thirteenth EuroSys Conference. pp. $1-15$ (2018)
[11] Baldimtsi, F., Camenisch, J., Dubovitskaya, M., Lysyanskaya, A., Reyzin, L., Samelin, K., Yakoubov, S.: Accumulators with applications to anonymity-preserving revocation. In: 2017 IEEE European Symposium on Security and Privacy (EuroS). pp. 301-315. IEEE (2017)

[12] Bano, S., Sonnino, A., Al-Bassam, M., Azouvi, S., McCorry, P., Meiklejohn, S., Danezis, G.: Sok: Consensus in the age of blockchains. In: Proceedings of the 1st ACM Conference on Advances in Financial Technologies. pp. 183-198 (2019)

[13] Baric, N., Pfitzmann, B.: Collision-free accumulators and fail-stop signature schemes without trees. Lecture Notes in Computer Science (including subseries Lecture Notes in Artificial Intelligence and Lecture Notes in Bioinformatics) 1233, 480-494 (1997)

[14] Ben-Sasson, E., Bentov, I., Horesh, Y., Riabzev, M.: Scalable, transparent, and post-quantum secure computational integrity. IACR Cryptol. ePrint Arch. p. 46 (2018)

[15] Ben-Sasson, E., Chiesa, A., Genkin, D., Tromer, E., Virza, M.: Snarks for c: Verifying program executions succinctly and in zero knowledge. In: Annual cryptology conference. pp. 90-108. Springer (2013)

[16] Benaloh, J., Mare, M.D.: One-Way Accumulators: A Decentralized Alternative to Digital Signatures (Extended Abstract). International Conference on the Theory and Applications of Cryptographic Techniques pp. 274-285 (1994)

[17] Boneh, D., Bünz, B., Fisch, B.: Batching Techniques for Accumulators with Applications to IOPs and Stateless Blockchains. Lecture Notes in Computer Science (including subseries Lecture Notes in Artificial Intelligence and Lecture Notes in Bioinformatics) pp. 561-586 (2019)

[18] Bünz, B., Bootle, J., Boneh, D., Poelstra, A., Wuille, P., Maxwell, G.: Bulletproofs: Short proofs for confidential transactions and more. In: 2018 IEEE Symposium on Security and Privacy (SP). pp. 315-334. IEEE (2018)

[19] Bünz, B., Kiffer, L., Luu, L., Zamani, M.: Flyclient: Super-light clients for cryptocurrencies. IACR Cryptology ePrint Archive 2019, 226 (2019), https://eprint.iacr.org/2019/226

[20] Cactus, H.: Hyperledger Cactus Whitepaper. Tech. rep. (2020), https: //github.com/hyperledger/cactus/blob/master/whitepaper/whitepaper.md

[21] Camenisch, J., Lysyanskaya, A.: Dynamic accumulators and application to efficient revocation of anonymous credentials. Lecture Notes in Computer Science (including subseries Lecture Notes in Artificial Intelligence and Lecture Notes in Bioinformatics) pp. 61-76 (2002)

[22] Castro, M., Liskov, B., et al.: Practical Byzantine fault tolerance. In: OSDI. vol. 99, pp. 173-186 (1999)

[23] Corda: Corda (2020), https://docs.corda.net/

[24] Desrosiers, L., Novotny, P., Ramakrishna, V.: Trade contracts (2020), https://github.com/HyperledgerHandsOn/trade-contracts

[25] Desrosiers, L., Novotny, P., Ramakrishna, V.: Trade network (2020), https://github.com/HyperledgerHandsOn/trade-network

[26] Everledger: Everledger — Tech for Good Blockchain Solutions (2020), https://www.everledger.io/

[27] Ferris, C.: Answering your questions on Hyperledger Fabric performance and scale (2019), https://www.ibm.com/blogs/blockchain/2019/01/ answering-your-questions-on-hyperledger-fabric-performance-andscale/

[28] Gervais, A., Karame, G.O., Gruber, D., Capkun, S., Zurich, E.: On the Privacy Provisions of Bloom Filters in Lightweight Bitcoin Clients. Tech. rep. (2014)

[29] Gudgeon, L., Moreno-Sanchez, P., Roos, S., McCorry, P., Gervais, A. Sok: Layer-two blockchain protocols. In: International Conference on Financial Cryptography and Data Security. pp. 201-226. Springer (2020)

[30] Hearn, M.: https://github.com/bitcoin/bips/blob/master/bip0037.mediawiki (2012), https://github.com/bitcoin/bips/blob/master/bip0037.mediawiki

[31] Hyperledger: Hyperledger Besu (2020), https://besu.hyperledger.org/

[32] Hyperledger: Hyperledger Caliper (2020), https://www.hyperledger.org/ use/caliper

[33] Hyperledger: Hyperledger Fabric docs (2020), https://hyperledgerfabric.readthedocs.io/en/latest/

[34] Hyperledger: Hyperledger Fabric gRPC Service Definitions (2020), https://hyperledger-fabric.readthedocs.io/en/latest/

[35] jolestar: Rsa accumulator (2019), https://github.com/westarlabs/rsaaccumulator

[36] Kalodner, H., Goldfeder, S., Chen, X., Weinberg, S.M., Felten, E.W.: Arbitrum: Scalable, private smart contracts. In: 27th \{USENIX\} Security Symposium (\{USENIX\} Security 18). pp. 1353-1370 (2018) 
[37] Kattis, A., Bonneau, J.: Proof of necessary work: Succinct state verification with fairness guarantees. IACR Cryptololgy ePrint Archive 2020, 190 (2020), https://eprint.iacr.org/2020/190

[38] Khalil, R., Gervais, A., Felley, G.: Nocust-a non-custodial 2nd-layer financial intermediary. IACR Cryptol. ePrint Arch. p. 642 (2018)

[39] Khalil, R., Zamyatin, A., Felley, G., Moreno-Sanchez, P., Gervais, A.: Commit-chains: Secure, scalable off-chain payments. Tech. rep., Cryptology ePrint Archive, Report 2018/642, 2018. https://eprint. iacr. org ... (2018)

[40] Kiayias, A., Lamprou, N., Stouka, A.P.: Proofs of proofs of work with sublinear complexity. In: International Conference on Financial Cryptography and Data Security. pp. 61-78. Springer (2016)

[41] Kiayias, A., Miller, A., Zindros, D.: Non-interactive proofs of proof-ofwork. IACR Cryptology ePrint Archive (963), 1-42 (2017)

[42] Komgo: Komgo (2020), https://komgo.io/

[43] Kwon, J., Buchman, E.: Cosmos whitepaper. Tech. rep., https://cosmos. network/resources/whitepaper

[44] Li, J., Li, N., Xue, R.: Universal accumulators with efficient nonmembership proofs. Lecture Notes in Computer Science (including subseries Lecture Notes in Artificial Intelligence and Lecture Notes in Bioinformatics) pp. 253-269 (2007)

[45] MarcoPolo: Marco Polo - A Trade Finance Initiative (2020), https:// www.marcopolo.finance/

[46] Matetic, S., Wüst, K., Schneider, M., Kostiainen, K., Karame, G., Capkun, S.: BITE: bitcoin lightweight client privacy using trusted execution. In: 28th USENIX Security Symposium 2019. pp. 783 800. USENIX Association (2019), https://www.usenix.org/conference/ usenixsecurity 19/presentation/matetic

[47] Matter Labs: Trustless scalability and privacy: Unchaining Ethereum with Zero-Knowledge Proofs, https://matter-labs.io

[48] McCorry, P., Bakshi, S., Bentov, I., Meiklejohn, S., Miller, A.: Pisa: Arbitration outsourcing for state channels. In: Proceedings of the 1 st ACM Conference on Advances in Financial Technologies. pp. 16-30 (2019)

[49] MultiChain: Multichain (2020), https://www.multichain.com/

[50] Nakamoto, S.: Bitcoin: A peer-to-peer electronic cash system. Tech. rep., Manubot (2019)

[51] Quorum: Quorum documentation (2020), http://docs.goquorum.com/en/ latest/

[52] Ripe.io: ripe.io (2020), https://www.ripe.io/

[53] Thakkar, P., Nathan, S., Viswanathan, B.: Performance benchmarking and optimizing hyperledger fabric blockchain platform. In: 2018 IEEE 26th International Symposium on Modeling, Analysis, and Simulation of Computer and Telecommunication Systems (MASCOTS). pp. 264-276. IEEE (2018)

[54] TradeLens: TradeLens - Digitizing global supply chains (2020), https: //www.tradelens.com/

[55] Trust, I.F.: IBM Food Trust - Blockchain for the world's food supply - IBM (2020), https://www.ibm.com/blockchain/solutions/food-trust

[56] WeTrade: We.Trade Trade Finance Network (2020), https://we-trade. com/

[57] Wood, G.: Polkadot: Vision for a heterogeneous multi-chain framework. White Paper (2016)

[58] Zhang, W., Yu, J., He, Q., Guan, N.: Tick: Tiny client for blockchains IACR Cryptology ePrint Archive (2019), https://eprint.iacr.org/2019/ 792.pdf

\section{APPENDIX A \\ RELATED WORK}

Light Clients. Verifying blockchain states without having full access to the ledger was envisioned in the original Bitcoin paper [50]. For instance, resource constrained light clients may need to confirm the inclusion of their transactions in the blockchain, but lack the resources to store and validate the entire ledger. Bitcoin leverages the Simplified Payment Verification (SPV) technique [50] to allow light clients to maintain only block headers and receive Merkle proofs from full nodes about transactions related to them. However, there are a number of limitations to this protocol. Light clients have to validate and store every block header, trust full nodes for transaction validation, and divulge significant information and cede privacy. There have been numerous efforts to address these limitations [40], [41], [19], [37], [58], [30], [28], [46]. Nonetheless, SPV-based schemes are not applicable to permissioned ledgers [33], [23], [31], [51], [49] as external clients do not have visibility on the ledger's history, including block headers and management policies.

Layer-2 Protocols. Layer-2 protocols are approaches to scaling that allow users to transact off-chain, enabling lower latencies, little or no fees and private transactions. Layer-2 protocols, sometimes referred to as commit-chains [39], are noncustodial and rely on the parent chain for security. Examples of these systems include Plasma [4], NOCUST [38], Optimistic Rollups schemes such Arbitrum [36] and ZK-Rollups schemes such as zkSync [47], [6]. These protocols checkpoint state at periodic intervals to Ethereum and rely on a challengeresponse game for integrity and/or data availability. In the case of Plasma or Optimistic Rollups users submit fraud proofs to a designated contract when fraud is detected. In the case of ZK-Rollups, consistency is inherent in the zero-knowledge proof construction, but operators are still challenged on data availability. Third-party watchers such as PISA [48] can augment Layer- 2 protocols by being incentivized to monitor the actions of operators. In contrast to the model in which Layer2 protocols operate, which includes non-custodial services with safe exits to the parent chain, permissioned networks are closed systems which create and manage their own state. External clients, including third-party watch services, are unable to monitor the actions of the network since no data is revealed for privacy and confidentiality reasons. Furthermore, the general purpose nature of these ledger technologies and their broad applications to real world problems makes using zero-knowledge schemes such as ZK-SNARKS [15], ZKSTARKS [14] and Bullet-Proofs [18] difficult and expensive.

Cross-Chain Communication Protocols. Protocols such as Cosmos [43], Polkadot [57], Cardano [2], Ren [5] and BTC Relay [1] enable communication of state, representing either data or assets, across different chains. These systems rely on an intermediate network of nodes in order to relay messages. In order to ensure that the state communicated is valid, these intermediaries require access to the internal state of the source chains (even if only block headers). Systems such as Cosmos and Polkadot also require chains to follow prescribed protocol specifications (either directly or through bridges) in order to participate in the ecosystem. However, the number of realworld deployments of permissioned ledgers that already exist make it impractical to migrate them into ecosystems like Cosmos and Polkadot. Furthermore, permissioned ledgers are closed sovereign networks that don't reveal any chain data externally, making them difficult to integrate.

Recently, efforts have emerged around relaying states across permissioned ledgers. Abebe et. al [7] and Hyperledger Cactus [20] describe systems for state sharing between independent permissioned networks by relaying attestations. While 
these systems have practical value, they rely on the external client placing significant trust on the committee members signing the state for its validity and currency. Our work, however, assumes a worst case scenario that requires only one honest member in the committee for providing guarantees on the validity of state while allowing clients to reason about its currency.

\section{APPENDIX B \\ BULLETIN BOARD}

Pseudocode of the bulletin board is presented in algorithm 1. The bulletin board records commitments from any management committee member, $m$, about the state of a permissioned ledger at height $i$ (in the form of a view $\mathcal{V}_{i}^{m}$ ), and its lineage thus far (in the form of a rolling hash $H_{i}^{m}$ ). Each time a commitment is submitted, the bulletin board checks that the value of the submitted commitment does not conflict with what has already been submitted by other members, and that the member's history of past accumulators matches the bulletin board's own up to that point. If either of these conditions fail, the bulletin board raises an event to notify all members of this inconsistency, as it indicates malicious behavior. The list of all members that have explicitly published a commitment for ledger height $i$ is stored, and their rolling hash is observed as an endorsement of past commitments. Using these, the bulletin board knows which past views have been explicitly and implicitly endorsed by all members of the committee. The bulletin board also provides a mechanism for committee members to report conflicts that they observe at any point. The bulletin board notifies all management committee members and subscribed external clients of events such as conflicts being detected and new commitments being published so as to ensure detectability and accountability of actions.

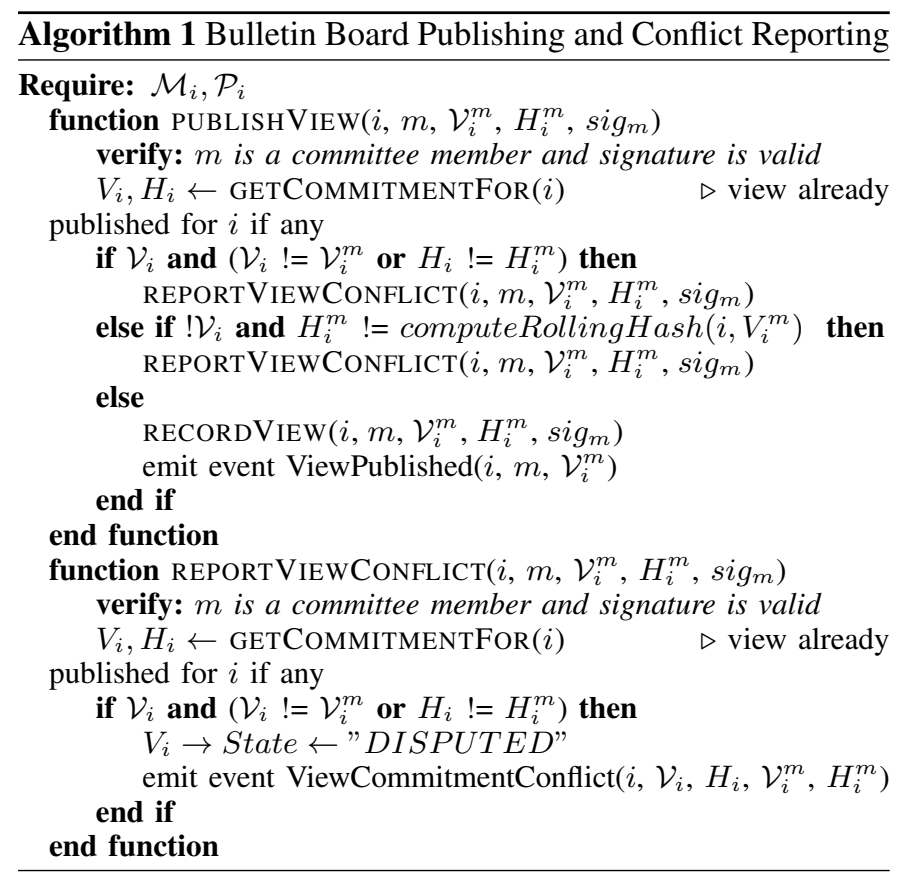

APPENDIX C

RSA ACCUMULATOR

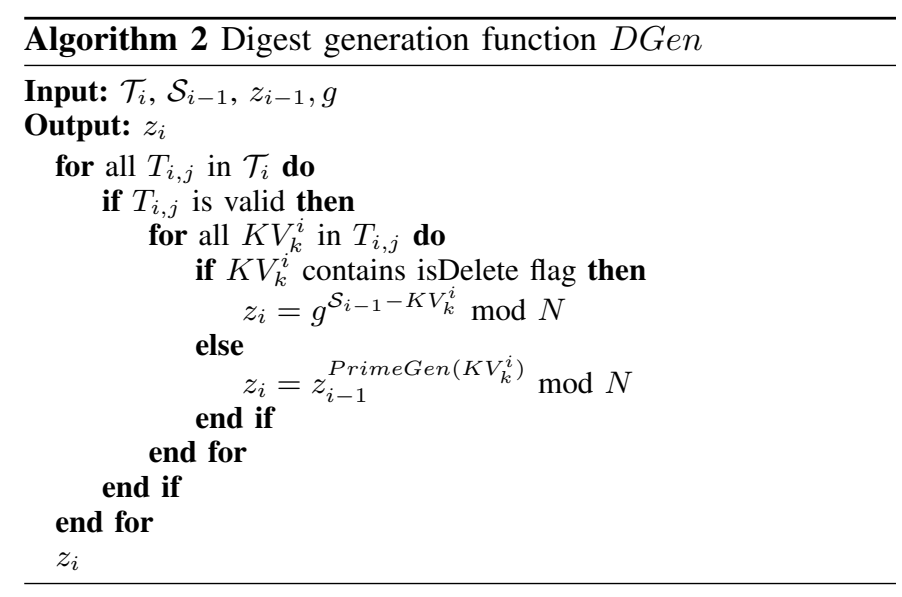

An RSA accumulator is a cryptographic primitive first described by Benaloh and de Mar [16]. It generates a succinct digest of a set of elements and allows for proof of membership of an element in the set without revealing any other information about the set. Variations of this accumulator to provide dynamic updates and both membership and nonmembership proofs were later proposed [21], [44], [11]. The accumulator is set up with an RSA modulus $N$ that is the product of two safe primes $p$ and $q$. A base generator $g$ is selected from the set of quadratic residues modulo $N$. The accumulated value for a set $X=\left\{x_{1}, \ldots, x_{n}\right\}$ is defined as $z=g^{x_{1} \ldots x_{n}} \bmod N$. Importantly, this set must consist of primes and therefore any non-primes must be converted to primes through a PrimeGen conversion function. In our implementation, the PrimeGen function hashes the element to be accumulated with an incremental nonce and checks the result for primality until a suitable nonce is found. However, there are other methods for prime conversion, for example in [13].

Adding a new element, $x^{\prime}$, to the accumulator is done by $z^{\prime}=z^{x^{\prime}} \bmod N$, where $z^{\prime}$ is the new accumulated value equivalent to $g^{X \cup\left\{x^{\prime}\right\}}$. Deleting element $x^{\prime}$ from the accumulator can be done efficiently by using auxiliary information about the accumulator, namely $\Phi(N)=(p-1)(q-1)$. Removal of $x_{j}$ from the accumulator using this method is done by $z^{\prime}=z^{x_{j}^{-1} \bmod \Phi(N)} \bmod N$, where $z^{\prime}$ is the accumulated value equivalent to $g^{X \backslash\left\{x_{j}\right\}}$.

A membership proof for an element $x_{j}$ in $z$ can similarly be generated using the accumulator's auxiliary information to calculate $z^{x_{j}^{-1} \bmod \Phi(N)}$. The consumer of the proof can verify it in constant time by checking that $w^{x_{j}} \bmod N=z$. A description of the generation of a non-membership proof that can be generated efficiently and verified in constant time is presented in [44].

The dynamic accumulator scheme also allows update of membership proofs in constant time. After adding a new element, $x^{\prime}$, update of the witness $w$ of element $x$ in $z$ to a witness that proves $x$ is a member of $z^{\prime}$ is done by $w^{\prime}=w^{x^{\prime}}$. 
After deletion of an element $x^{\prime}$, the witness $w$ of $x$ in $z$ (where $x \neq x^{\prime}$ ) can be updated to prove $x$ in $z^{\prime}$ by $w^{\prime}=g^{b} z^{\prime a}$, where $a$ and $b$ are integers computed through the extended Euclid algorithm such that $a x+b x^{\prime}=1$. The efficient update of a non-membership witness is described in [44].

As the elements included in the accumulator must be primes, every key must find a suitable nonce such that the resulting hash of the key and its nonce is prime. Finding this nonce can be done in a deterministic fashion. However, finding this nonce takes some computational effort and therefore the RSA accumulator in our implementation stores a map of all the accumulated keys and their nonces. This enables fast lookup of whether an element is in the accumulated set and the ability to create the accumulator after deletion of a key from the ledger, without having to retrieve all state from the peer's ledger.

\section{APPENDIX D \\ PROOF OF THEOREM 1}

Theorem 1: Assuming the strong RSA assumption, a secure signature scheme, and the security of the bulletin board, the permissioned ledger $\mathcal{L}^{n}$ of the proposed protocol is Type-2 secure.

Proof: We prove this by contradiction. Assume that the system is not Type 2 secure. Then, according to Definition 10, either of the following two conditions hold: (1) there exists $F \in \mathcal{F}^{*}, W \in \mathcal{W}^{*}, V \in \mathcal{V}^{*}$, such that the system is not Type1 secure; or (2) there exists $F \in \mathcal{F} \backslash \mathcal{F}^{*}, W \in \mathcal{W} \backslash \mathcal{W}^{*}, V_{i} \in$ $\mathcal{V} \backslash \mathcal{V}^{*}$, such that $\operatorname{WPVer}\left(F, W, V_{i}\right)=$ True, but there does not exist $V_{i}^{\prime} \in V^{*}$ or $A \in\{I, E, M\}$, s.t. external view $V_{i}$ is not consistent with $V_{i}^{\prime}$ or $K_{A}^{H}\left(V_{i}, V_{i}^{\prime}\right)$ does not hold.

We first prove condition (2) by contradiction. For all $F \in$ $\mathcal{F} \backslash \mathcal{F}^{*}, W \in \mathcal{W} \backslash \mathcal{W}^{*}, V_{i} \in \mathcal{V} \backslash \mathcal{V}^{*}$, if $\operatorname{WPVer}\left(F, W, V_{i}\right)=$ True and the system is not Type- 1 secure, then according to Definition 9, either of the following three subconditions does not hold:

1) $\operatorname{PGen}\left(F, \mathcal{D}_{i}, \mathcal{M}_{i}, \mathcal{P}_{i}\right)=\pi$

2) $\operatorname{VGen}\left(\mathcal{D}_{i}, \mathcal{M}_{i}, \mathcal{P}_{i}\right)=V_{i}$

3) $\mathrm{WPGen}\left(F, \pi, \mathcal{D}_{i}, V_{i}\right)=W$

If the subcondition 1, i.e., $\operatorname{PGen}\left(F, \mathcal{D}_{i}, \mathcal{M}_{i}, \mathcal{P}_{i}\right)=$ $\pi$, does not hold, then there exists a proof $\pi^{\prime}$ such that $\operatorname{PVer}\left(F, \pi^{\prime}, \mathcal{D}_{i}\right)=$ True and there does not exist $\operatorname{PGen}\left(F, \mathcal{D}_{i}, \mathcal{M}_{i}, \mathcal{P}_{i}\right)=\pi^{\prime}$ for some $F, \mathcal{D}_{i}$, and $\mathcal{M}_{i}$. Assuming the strong RSA assumption, this contradicts the soundness of the RSA accumulator, due to Theorem 2 of [21].

If subcondition 2, i.e., $\operatorname{VGen}\left(\mathcal{D}_{i}, \mathcal{M}_{i}, \mathcal{P}_{i}\right)=V_{i}$, does not hold, then the view $V$ is a fake view for an external party. In other words, $\left(\mathcal{D}_{i}, \mathcal{M}_{i}, \mathcal{P}_{i}\right)$ does not represent the system state $\mathcal{S}_{i}$. One condition for the validity of the view is the wrapped view must be present in the bulletin board. As we will analyse the wrapping process in subcondition 3, let's assume that the wrapping process is secure. In addition, as we assume the security of the underlying bulletin board, any party has access to the immutable record of it. According to our security model, there exists at least one honest party which has access to the bulletin board. Thus, this party will have the knowledge of this fake view represented by
$\left(\mathcal{D}_{i}, \mathcal{M}_{i}, \mathcal{P}_{i}\right)$ such that $\operatorname{VVer}\left(\mathcal{D}_{i}, \mathcal{M}_{i}, \mathcal{P}_{i}, V_{i}\right)=$ False, and the corresponding correct view $V_{i}^{\prime}$. So, $K_{A}^{H}\left(V_{i}, V_{i}^{\prime}\right)$ would hold such that external view $V_{i}$ is not consistent with $V_{i}^{\prime}$. This is evidence of misbehavior.

If subcondition 3, i.e., WPGen $\left(F, \pi, \mathcal{D}_{i}, V_{i}\right)=W$ does not hold, then either the proof $\pi$ is forged, or the view $V_{i}$ is forged, or the verification $\operatorname{WPVer}\left(F, W, V_{i}\right)$ is not sound. The first two subconditions are already proved. For the last one, as the wrapping generation and verification in our proposed scheme is done by using a secure signature scheme. If $\operatorname{WPVer}\left(F, W, V_{i}\right)=$ True and $\operatorname{WPGen}\left(F, \pi, \mathcal{D}_{i}, V_{i}\right)=W$ is not true, then this contracts to the unforgeability of the signature scheme. Thus, this contradicts the assumption of a secure signature scheme.

With the above proofs w.r.t. the three subconditions, condition (2) does not hold by contraction. Condition (1) has similar subconditions that are required for proving Condition (2), and the proof is similar to the verification of condition (2). 